\title{
Baldrian-Hopfen-Kombination verkürzt Einschlafzeit
}

— Verlängerte Einschlafzeiten und nicht ausreichend erholsamer Schlaf können unbehandelt zu Leistungseinbußen und zu verringerter Lebensqualität führen. Sobald die Einschlafdauer über einen längeren Zeitraum 30 Minuten übersteigt, ist von einer Einschlafstörung zu sprechen.

Wenn keine organisch oder medikamentös bedingte Ursache zugrunde liegt, kann der Spezialextrakt Ze91019 aus Baldrianwurzel und Hopfenzapfen (Allunapret ${ }^{\circledR}$ ) die Länge der Einschlafzeiten signifikant verkürzen.
Dies zeigte eine klinische Studie mit $30 \mathrm{Er}$ wachsenen, deren Einschlafzeit vor Studienbeginn über 30 Minuten lag [1]. Verglichen wurde der Baldrian-Hopfen-Spezialextrakt mit einer Baldrian-Monotherapie und Placebo. Nach vier Wochen zeigte sich eine Senkung der Einschlafzeit um 22 Minuten unter der Baldrian-Monotherapie und um $45 \mathrm{Mi}$ nuten unter der Baldrian-Hopfen-Kombination. Damit war die Einschlafzeit nur unter der Kombination signifikant gegenüber Placebo verkürzt.
Das pflanzliche Kombinationspräparat ist gut verträglich. Nebenwirkungen, Hangover oder eine Abhängigkeit sind nicht bekannt. Erste spürbare Verbesserungen treten bereits in den ersten Tagen der Therapie auf. Da das Präparat aber schonend in die Schlafregulation eingreift, entfaltet sich die volle Wirksamkeit erst nach ein bis zwei Wochen.

- Red.

Quellen: 1. U. Koetter et al. Phytother Res 2007; 21 (9):847-51; Presseinformationen von Bionorica SE

\section{NO fördert Ausbildung von Kollateralgefäßen \\ Gegen Durchblutungsstörungen antrainieren}

- Bei arteriellen Durchblutungsstörungen sollte das Gefäßtraining - neben der Kontrolle von Risikofaktoren sowie interventioneller und medikamentöser Therapien nicht unterschätzt werden. Auch für die Ausbildung körpereigener Umgehungskreisläufe ist körperliche Bewegung und die damit verbundene Stickoxid-(NO-)Freisetzung von großer Bedeutung.

Was körperliches Training bewirken kann, wurde u. a. in der PET-Studie (PTCA \& Exercise Training) gezeigt. 101 Patienten mit stabiler KHK erhielten hier neben der medikamentösen Standardtherapie entweder eine interventionelle Revaskularisation oder sie betrieben jeden Tag 20 min Sport bei $70 \%$ der maximalen Belastung. Nach einem Jahr war die Rate an kardiovaskulären Ereignissen (Herzinfarkt, Schlaganfall, Revaskularisation oder Hospitalisierung wegen Angina pectoris) in der Trainingsgruppe signifkant geringer als in der Stentgruppe (12\% vs. $30 \%)$. Dieser Vorteil ließ sich auch noch nach fünf Jahren nachweisen (s. Abb.). In einer weiteren Studie wurde nachgewiesen, dass auch Patienten, bei denen wegen einer peripheren arteriellen Verschlusskrank- heit mit Claudicatio intermittens eine PTCA durchgeführt wurde, von einem körperlichen Training profitieren. Bei der Kombination beider Maßnahmen war die Gehstrecke deutlich länger als nach Revaskularisation oder Training allein.

\section{Bewegung bessert endotheliale Dysfunktion}

Für den positiven Effekt des körperlichen Trainings gibt es zwei Erklärungen. Zum einen wurde gezeigt, dass eine Bewegungstherapie die endotheliale Dysfunktion und damit die NO-Freisetzung fördert. NO ist bekanntlich ein starker Vasodilatator, senkt daher die Vor- und Nachlast des Herzens und verbessert die Perfusion des Myokards, erklärte Priv.-Doz. Ivo R. Buschmann, Berlin.

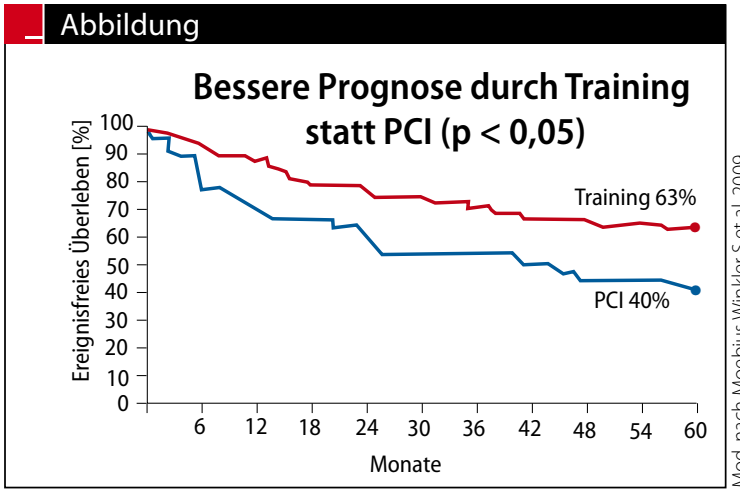

Stabile KHK: Training kann oft mehr bewirken als eine PCI.
Wachstum „biologischer Bypässe“

Ein weiterer Mechanismus ist die Ausbildung von „biologischen Bypässen“, erklärte Buschmann. Jeder Mensch hat im Sinne einer "Vierten Zirkulation" bereits angelegte "Umgehungsstraßen", die bei Bedarf aktiviert werden können. Dies erklärt, warum es Patienten gibt, die trotz Stenosen von Hauptgefäßen keine Beschwerden haben. Neuere Untersuchungen zeigten, dass NO ein Schlüsselmolekül bei der Induktion von Proliferation und Wachstum dieser Gefäße ist. Durch die Scherkräfte bei körperlicher Bewegung und den erhöhten pulsatilen Blutfluss wird immer wieder kurzfristig NO freigesetzt, was ein wesentlicher Wachstumsreiz für die korkenzieherähnlichen Kollateralgefäße zu sein scheint.

Zur Zeit wird an der Charité untersucht, ob man diesen Effekt auf die KollateralienAusbildung auch durch die intermittierende Gabe eines NO-Donators wie Nitroglycerin erreichen kann. Wenn sich dies bestätigt, hätte man in der Optimierung des Gefäßtrainings-Effektes eine wichtige neue Indikation für ein kurz wirksames Nitrat (wie z. B. Nitrolingual ${ }^{\circledR}$ akut Spray), meinte Buschmann.

- Maria Weiß

Quelle: Satellitensymposium auf der Jahrestagung der Dtsch. Gesellschaft für Prävention und Rehabilitation von Herz-Kreislauferkrankungen, Berlin, Juni 2011 (unterstützt von Pohl Boskamp) 